\title{
Surgery for combined type small cell lung carcinoma
}

\author{
R Hage, J R J Elbers, A Brutel de la Rivière, J M M van den Bosch
}

\begin{abstract}
Background-Combined type small cell lung cancer (SCLC) has been reported to occur in, at most, $1 \%$ of all cases of SCLC. These tumours consist of SCLC with a component of squamous cell carcinoma and/or adenocarcinoma. The survival of patients with combined and pure SCLC after surgical resection was assessed.

Methods-From 1977 to 19942115 patients with bronchogenic carcinoma underwent pulmonary resection. From this group 26 patients $(1.2 \%)$ were diagnosed as having combined SCLC and 74 patients $(3.5 \%)$ as having pure SCLC.
\end{abstract}

Results-From the 26 patients with combined SCLC (mean age 66.4 years) three were classified as pT1N0M0, eight as pT2N0M0, four as postoperative stage II, and 11 as postoperative stage III. Histological examination showed a component of squamous cell carcinoma in 21 patients. There were 18 (69\%) lobectomies, seven (27\%) pneumonectomies, and one (4\%) segmentectomy. In all patients surgery was thought to be curative. Overall hospital mortality was $4 \%(n=1)$. Cumulative five year survival was $31 \%$ for all hospital survivors with combined SCLC postoperative stage $I, 50 \%$ for those with pT1N0M0, and $25 \%$ for those with pT2N0M0 disease. No patients with postoperative stage II and III disease survived for five years. In the $\mathbf{7 4}$ patients with pure SCLC hospital mortality was $3 \%(n=2)$; cumulative five year survival was $39 \%$ in patients with postoperative stage I disease, $46 \%$ for those with pT1N0M0 and $35 \%$ for those with pT2N0M0. When compared with pure SCLC, no significant differences in five year survival were evident in patients with postoperative stage I disease.

Conclusions-Surgical resection in patients with combined SCLC postoperative stage I yields a cumulative five year survival of $31 \%$ while for those with stage II and III disease there were no survivors at five years. In patients with stage I combined or pure SCLC surgery can offer a long term disease free interval or may even be curative.

(Thorax 1998;53:450-453)

Keywords: lung cancer; surgery; histological subtypes

Received 30 June 1997 Returned to authors 18 December 1997 Revised version received 5 January 1998

Accepted for publication

7 January 1998
According to the WHO criteria with modifications by the Pathology Committee of the International Association for the Study of Lung
Cancer (IASLC), three subtypes of small cell lung carcinoma (SCLC) have been recognised: pure ("classic"), mixed, and combined.

Mixed SCLC are tumours with a mixture of both SCLC and large cell carcinoma. Combined type SCLC has been estimated to contain, at most, $1 \%$ of all types of chemonaive SCLC. ${ }^{1}$ These tumours, as defined by the IASLC, are SCLC admixed with a component of squamous cell carcinoma and/or adenocarcinoma.

To our knowledge, few data with very small numbers of patients are available regarding the prognosis and clinical behaviour of patients with combined type SCLC. ${ }^{1-6}$ In this study we have investigated retrospectively the five year survival after surgical resection in 26 patients with combined type SCLC and 74 patients with pure SCLC and have compared the prognosis for patients in these two groups.

\section{Methods}

From 1977 to 19942115 patients with bronchogenic carcinoma underwent pulmonary resection at the St Antonius Hospital, Nieuwegein, The Netherlands.

The histology of all the surgical resection specimens was reviewed. Diagnosis was based on light microscopic criteria according to the consensus report by the IASLC ${ }^{1}$ In nearly all cases the diagnosis made by light microscopy was confirmed by immunohistochemical methods.

Both the preoperative clinical and postoperative pathological TNM classifications ${ }^{7}$ were reported for all patients. Clinical TNM staging included routine physical examination, radiological chest imaging techniques, haematological and blood chemistry examination, as well as bronchoscopy and mediastinoscopy.

COMBINED SCLC

Combined SCLC was diagnosed in 26 patients $(1 \% ; 25$ men) of mean age 66.4 years (range 53-76). The preoperative diagnosis was nonsmall cell lung carcinoma (NSCLC) in 14 patients, unknown in eight, pure SCLC in three, and undifferentiated carcinoma suspected as SCLC in one patient.

In the three patients in whom SCLC was suspected one was staged as CT1N0 and two as cT2N0. In the first patient surgery was performed because, due to his age (72 years), chemotherapy was thought not to be the treatment of first choice. In the other patient pneumonectomy was potentially curative. The third patient was treated by neoadjuvant chemotherapy with CDE (cyclophosphamide, doxorubicin, etoposide) resulting in stable disease. 
Table 1 Number of patients in pretreatment clinical and postoperative TNM stage for combined and pure small cell lung cancer (SCLC)

\begin{tabular}{|c|c|c|c|c|}
\hline & \multicolumn{2}{|c|}{ Combined SCLC } & \multicolumn{2}{|c|}{ Pure SCLC } \\
\hline & $c T N M$ & $p T N M$ & $c T N M$ & $p T N M$ \\
\hline Stage 0 & NA & 0 & NA & 3 \\
\hline Stage I & 23 & 11 & 70 & 33 \\
\hline T1N0 & 7 & 3 & 25 & 13 \\
\hline T2N0 & 16 & 8 & 45 & 20 \\
\hline Stage II & 0 & 4 & 0 & 13 \\
\hline Stage III & 3 & 11 & 4 & 25 \\
\hline
\end{tabular}

$\mathrm{NA}=$ not applicable

Table 2 Five year survival (\%) in pretreatment clinical and postoperative TNM stage for combined and pure small cell lung cancer (SCLC)

\begin{tabular}{|c|c|c|c|c|}
\hline & \multicolumn{2}{|c|}{ Combined SCLC } & \multicolumn{2}{|c|}{ Pure SCLC } \\
\hline & $c T N M$ & $p T N M$ & $c T N M$ & $p T N M$ \\
\hline Stage I & 15 & 31 & 25 & 39 \\
\hline T1N0 & 29 & 50 & 32 & 46 \\
\hline T2N0 & 8 & 25 & 21 & 35 \\
\hline Stage II & NA & 0 & NA & 8 \\
\hline Stage III & 0 & 0 & 0 & 17 \\
\hline
\end{tabular}

$\mathrm{NA}=$ not applicable; all percentages rounded to nearest whole number.

Because there was no evidence of distant metastases, and mediastinoscopy was negative, pneumonectomy was performed.

The patient in whom the preoperative diagnosis was undifferentiated carcinoma suspected as being SCLC underwent lobectomy. There was no evidence of metastatic disease.

Mediastinoscopy was negative in all but two patients, one with $\mathrm{N} 2$ disease and the other with $\mathrm{N} 3$ disease. The patient with $\mathrm{N} 2$ disease was initially diagnosed as having non-small cell carcinoma stage IIIA (N2) for which she received neoadjuvant MIC chemotherapy (mitomycin $\mathrm{C}$, ifosfamide and cisplatin) followed by lobectomy. Postoperative histopathological examination showed a combined SCLC. The patient with $\mathrm{N} 3$ disease (T2N3) underwent pneumonectomy with postoperative irradiation of 3900 cGy.

Eleven patients underwent surgery without any (neo-)adjuvant therapy. Surgical resection was preceded by chemotherapy in two patients while 13 patients received adjuvant chemotherapy. Due to the long study period chemotherapy protocols were not the same for all patients. All but two surgical resection specimens of patients with combined SCLC were chemonaive.

PURE SCLC

Pure SCLC was diagnosed in 74 patients $(3.5 \%$; 63 men) of mean age 63.6 years (range 45-84). The preoperative diagnosis was SCLC in 42 patients; in the other patients no preoperative diagnosis could be established ( $\mathrm{n}$ $=14$ ) or NSCLC was suspected $(\mathrm{n}=18)$. Of the 42 patients in whom the preoperative diagnosis was SCLC, 13 were classified as CT1N0, 27 as cT2N0, one as cT3N0, and one cT1N2.

Mediastinoscopy was negative in all patients except one who was treated by adjuvant chemotherapy with cyclophosphamide, methotrexate, and cyclohexylchloroethylnitrosourea (CMC).

Forty two patients $(57 \%)$ were treated with chemotherapy after surgery, seven (9.5\%) underwent neoadjuvant chemotherapy alone, and $10(13.5 \%)$ were treated with both neoadjuvant and adjuvant chemotherapy. Fifteen patients $(20 \%)$ received surgical treatment alone without chemotherapy. In this group of patients none was treated by irradiation.

ANALYSIS OF DATA

Kaplan-Meier survival analysis was performed on all hospital survivors and survival curves were constructed according to the KaplanMeier method. ${ }^{8}$ Differences in survival between combined and pure SCLC were tested by the log rank test for statistical significance. ${ }^{9}$

\section{Results}

COMBINED SCLC

According to the clinical TNM classification 23 patients were classified as cTNM stage I (seven cT1N0, 16 cT2N0) and three as cTNM stage III (one cT2N2, one cT3N2, one cT2N3). Seven patients (27\%) required pneumonectomy, eighteen patients $(69 \%)$ underwent lobectomy, while one patient underwent segmentectomy. In all patients surgery was thought to be curative with negative resection margins. The overall hospital mortality was $4 \%$ $(\mathrm{n}=1)$.

According to the pTNM classification 11 (42\%) patients had postoperative stage I disease, four (15\%) stage II, and $11(42 \%)$ stage III (table 1).

Postoperative histological examination showed a squamous cell carcinoma component in 21 patients, while in four patients an adenocarcinoma component was present. One patient had both adeno and squamous cell components.

Cumulative five year survival for all hospital survivors with postoperative stage I disease was $31 \%$. The five year survival according to the cTNM and pTNM classification is shown in table 2 .

PURE SCLC

Seventy patients were classified as cTNM stage I (25 cT1 and 45 cT2), four patients as cTNM stage III (one cT1N2 and three T3N0). In patients with pure SCLC there were 32 lobectomies, 27 pneumonectomies, 10 bilobectomies, and five segmentectomies.

Postoperative staging classified 33 patients as having pTNM stage I disease, 13 as having stage II disease and 25 patients stage III disease. In one patient with cT2N0 no tumour could be detected after induction chemotherapy. In two patients after induction chemotherapy no tumour was found.

The cumulative five year survival for patients with pure SCLC in postoperative stage I was $39 \%$. There was no significant difference in the cumulative five year survival between patients with postoperative stage I combined or pure SCLC ( $p=0.629)$, nor was there a significant difference in survival between patients with combined and pure SCLC in stages II and III. The survival curves according to the KaplanMeier method are shown in fig 1. 


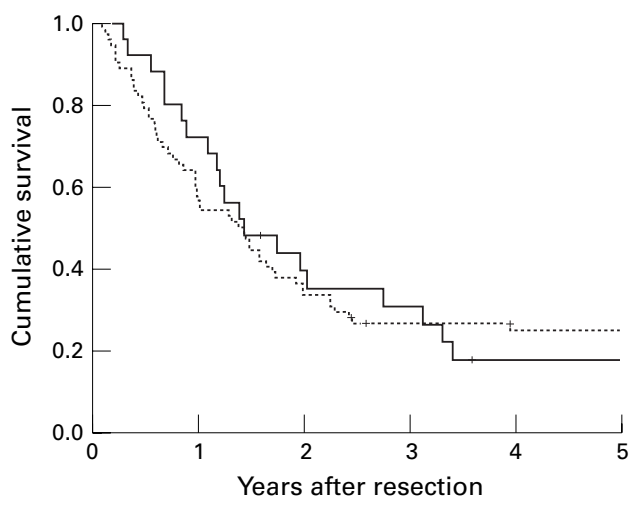

Figure 1 Cumulative five year survival curves of the hospital survivors according to the Kaplan-Meier method for combined (-) and pure (.....) SCLC.

\section{Discussion}

The spectrum of pulmonary neuroendocrine tumours is wide, ranging from the carcinoids which are low grade malignant tumours comprising $1-2 \%$ of all lung tumours to the most poorly differentiated lung tumours, the SCLC and the large cell neuroendocrine carcinomas (LCNEC), which carry a much worse prognosis. Within the spectrum of neuroendocrine tumours it is unknown whether SCLC, with more differentiated elements such as squamous or adenocarcinoma components, has a comparable prognosis to pure SCLC. In other words, does the presence of squamous or adenocarcinoma intermixed with SCLC alter the prognosis significantly?

Cumulative five year survival after surgical treatment was $39 \%$ for patients with stage I pure SCLC and $31 \%$ for those with stage I combined SCLC. In this series no significant differences in cumulative five year survival were found in patients with stage I combined SCLC and those with stage I pure SCLC. Also, in patients with clinical or postoperative stage II and III disease there were no significant differences in survival between combined and pure SCLC. Mangum et $a l^{\beta}$ reported nine patients with combined SCLC and also concluded that combined SCLC is clinically similar to pure SCLC.

Many histopathological classifications of SCLC subtypes have been proposed during the last 30 years. However, combined type SCLC as a separate category was proposed for the first time by the WHO in $1981 . .^{10}$ SCLC was then subdivided into oat cell type, intermediate type, and combined type. After these WHO criteria were modified by the IASCL ${ }^{11}$ the distinction between oat cell type and intermediate type was discarded and both categories were referred to as small cell carcinoma (SCLC). In addition, a new category-the mixed small cell/large cell carcinoma-was introduced as a subtype of SCLC. Since then this WHO classification, modified by the IASLC, has been the most generally accepted classification for small cell lung cancer.

Combined SCLC contains a squamous cell and/or adenocarcinoma component. The incidence of combined SCLC has been reported to range from less than $1 \%^{1}$ to $14.6 \%$ of all SCLC. ${ }^{1-36^{12}}$ In our series of 2115 patients with bronchogenic carcinoma who underwent pulmonary resection we found that 26 patients $(1.2 \%)$ had combined SCLC. Diagnosis was made using light microscopic criteria on surgical resection specimens. In none of the patients was the correct diagnosis of combined SCLC established before operation. As Fraire et al has suggested, the true frequency of combined type may thus be influenced by small or crushed biopsy samples, the number of histological slides studied, and whether or not surgical (or necropsy) specimens are available.

In clinical practice of pulmonary oncology it is important to know whether combined SCLC behaves clinically like a pure SCLC or if the non-small cell component largely determines the tumour characteristics. Because of the high chemosensitivity of pure SCLC, chemotherapy with or without radiotherapy achieves high response rates. However, in limited disease SCLC two year and five year survival is less than $15 \%$ and $10 \%$, respectively, because most patients will develop local or distant metastases when only treated with chemotherapy, with or without chest irradiation.

The surgical management of patients with SCLC is still controversial, and the role of surgery for SCLC has declined since the Medical Research Council trial reported the results of its 10 year follow up in $1973 .{ }^{13}$ This prospective trial concluded that radiotherapy resulted in better median survival and slightly better long term survival than surgery. Radiotherapy has since been replaced by chemotherapy as the cornerstone of treatment of SCLC. Studies by the Veterans Administration Surgical Oncology Group (VASOG) have again raised interest in the use of surgical resection with adjuvant chemotherapy for a small subset of patients with limited disease SCLC. ${ }^{14}$

Taking the cumulative five year survival rates after surgery into account, we conclude that surgical resection in selected patients with pretreatment clinical stage I combined and pure SCLC can be curative or offer long term survival. Smit et $a l^{15}$ also concluded that curative resection in highly selected patients with pure SCLC offers the best chance for long term survival.

The most appropriate combined modality treatment for both combined and pure SCLC seems to be resection with (neo-)adjuvant chemotherapy. However, the role of adjuvant chemotherapy in stage I SCLC remains to be determined. Prasad et $a l^{16}$ reported no significant difference in the cumulative five year survival in patients with stage I and II SCLC when chemotherapy followed surgical resection, compared with surgical resection alone. Karrer et $a l,{ }^{17}$ on the other hand, suggested that surgery followed by chemotherapy is an appropriate therapeutic approach for patients with SCLC stage I, II and T3 N0-1. Differences in chemotherapy between studies hamper proper comparison. In order to control possible micrometastases of the small cell component, and because of the high chemosensitivity of SCLC, combined modality treatment with chemotherapy should be appropriate. Only a limited number of our patients received neoadjuvant 
chemotherapy because a diagnosis of combined SCLC is difficult, if not impossible, to obtain from bronchoscopic biopsy specimens. Also, the number of patients with resectable combined SCLC is limited.

We had no patients with clinical stage II disease and only a few patients had stage III disease. As no significant long term survival in patients with stage III disease has been reported in most studies, ${ }^{16}$ these patients should be excluded from surgery.

Until recently the numbers of patients studied with combined SCLC have lacked convincing statistical power. To our knowledge we have described the largest series of patients with combined SCLC. However, a large multicentre study will be necessary to determine further the clinical behaviour and prognosis in patients with combined SCLC.

In conclusion, combined and pure SCLC behave in a clinically similar way. Surgical resection in highly selected patients with clinical pretreatment stage I SCLC should be considered because of the relatively high chance of cure.

The effects of (neo-)adjuvant chemotherapy remain inconclusive but we speculate that this could reduce the incidence of distant metastases due to micrometastases before or after radical surgical resection.

1 Hirsch FR, Matthews MJ, Aisner S, et al. Histopathologic classification of small cell lung cancer. Changing concepts and terminology. Cancer 1988;62:973-7.
2 Fraire $\mathrm{AE}$, Johnson $\mathrm{EH}$, Yesner $\mathrm{R}$, et al. Prognostic significance of histopathologic subtype and stage in small cell lung cancer. Hum Pathol 1992;23:520-8.

3 Mangum MD, Greco FA, Hainsworth JD, et al. Combined small-cell and non-small-cell lung cancer. $f$ Clin Oncol 1989;7:607-12.

4 Sehested M, Hirsch FR, Østerlind K, et al. Morphologic variations of small cell lung cancer. A histopathologic study of pretreatment and posttreatment specimens in 104 of pretreatment and posttreat.

5 Yesner R, Auerbach O, Gerstl B. Evolution of small cell carcinoma of lung. Chest 1979;76:A370.

6 Brereton HD, Mathews MM, Costa J, et al. Mixed anaplastic small-cell and squamous-cell carcinoma of the lung. Ann Intern Med 1978;88:805-6.

7 Mountain CF. A new international staging system for lung cancer. Chest 1986;89:225-33s.

8 Kaplan EL, Meier P. Non-parametric estimation from incomplete observations. F Am Stat Assoc 1958;53:457-81.

9 Peto R, Peto J. Asymptotically efficient rank invariant test procedures. F R Stat Soc (Series A) 1972;135:185-98.

10 The World Health Organization histological typing of lung tumours. 2nd edn. Am f Clin Pathol 1982;77:123-136.

11 Yesner R. Classification of lung cancer histology. $N$ Engl f Med 1985;312:652-3.

12 Hirsch FR, Ottesen G, Pødenphant J, et al. Tumor heterogeneity in lung cancer based on light microscopic features. Virchows Arch [A] 1983;402:147-53.

13 Fox W, Scadding JG. Medical Research Council comparative trial of surgery and radiotherapy for primary treatment of small-celled or oat-celled carcinoma of bronchus. Ten-year follow-up. Lancet 1973;ii:63-5.

14 Shields TW, Higgins GA, Matthews MJ, et al. Surgical resection in the management of small cell carcinoma of the lung. F Thorac Cardiovasc Surg 1982;84:481-8.

15 Smit EF, Groen HJM, Timens W, et al. Surgical resection for small cell carcinoma of the lung: a retrospective study. Thorax 1994;49:20-2.

16 Prasad US, Naylor AR, Walker WS, et al. Long term survival after pulmonary resection for small cell carcinoma of the lung. Thorax 1989;44:784-7.

17 Karrer K, Shield TW, Denck H, et al. The importance of surgical and multimodality treatment for small cell bronchial carcinoma. F Thorac Cardiovasc Surg 1989;97: 168-76. 